\title{
PHOTOMETRIC STEREO ASSISTED DRAWING OF ARCHITECTURAL RELIEFS
}

\author{
A. Abzal ${ }^{1 *}$, M. Saadatseresht ${ }^{1}$, M. Varshosaz ${ }^{2}$ \\ ${ }^{1}$ School of Surveying and Geospatial Engineering, College of Engineering, University of Tehran, Tehran; ali.abzal@ut.ac.ir \\ ${ }^{2}$ Geomatics Engineering College, K. N. Toosi University of Technology, Tehran, Iran, varshosazm@kntu.ac.ir
}

\author{
Commission VI, WG VI/4
}

KEY WORDS: Line Drawing, Geometric Documentation, Photometric Stereo, Fringe Projection

\begin{abstract}
:
Geometric documentation is one of the most important parts of a documentary report. Despite the advances made in the field of line drawing of ancient relief surfaces, in most cases, human operator interaction is unavoidable. In this paper, an algorithm for the semiautomatic line drawing of relief surfaces has been developed. In the proposed method, photometric stereo normals are used as a highresolution and low-noise data for the automatic extraction of surface edges. The normals are computed in $2 \mathrm{D}$ image space and also the fringe projection scanner is used for geometric correction of 2D image based drawings. Therefore, the drawings are converted to a metric map for geometric documentation reports. The results show that the efficiency of the proposed method, which has managed to correctly draw about more than $99 \%$ of the edge lines of an ancient relief. Also all of the drawn lines are completely coincided to the relief edges on its orthophoto image.
\end{abstract}

\section{INTRODUCTION}

Today, automatic drawing of reliefs is an important part of cultural heritage digital documentation. This data is an essential part of various activities including perception enhancement, preservation and restoration, monitoring and interpretation (Pintus, Pal et al. 2014). The objects modelled are usually complex and, having sharp varying depths, include naturally or artificially damaged parts or weak textures. As a result, the digitisation requires high resolution low noise techniques which are able to extract details. An example is that developed by (Karkınlı, Civicioglu et al. 2016). To draw lines of a relief, (Karkınl, Civicioglu et al. 2016) extracted the line features of a range image which was created from a point cloud obtained using dense matching. Despite having a good network accuracy, this technique is very dependent on the object texture. As a result, when the relief surface has a weak texture, the edges become noisy and, consequently, the line drawing becomes problematic. Also a variety of approaches are focused on 3D line extraction from dense point cloud. However, noisy points around edges, discrete data points and the complexity of topology definition in 3D data make it difficult to extract lines from point cloud (Goesele, Fuchs et al. 2003, Morita, Yokoyama et al. 2003). Therefore, it is necessary to enrich the point cloud with a high resolution-low noise data.

In this paper, a hybrid 3D scanner is proposed which comprises Photometric stereo (PS) method and fringe projection (FP) technique. The PS component is used to provide thematic information for high quality line drawing while the FP technique is used to acquire geometry needed to represent the drawings in a map. The usage of PS normal as a high resolution (Lu, Tai et al. 2013) and low high-frequency noise data (Nehab, Rusinkiewicz et al. 2005) allows to establish an automatic line drawing algorithm for complex relief surfaces. In this algorithm all of the drawings are done in a 2D image space and has minimum computational complexity for topology definition.
And also in contrast with the texture images, only geometric edges are represented in PS normals.

This paper includes four sections. After the introduction, the suggested algorithm is explained. Having discussed the general components of the system and the required elements for the algorithm to work properly, the steps taken to perform semiautomatic drawings are explained. Then, in Section 3, the system is examined numerically and visually to show how capable it is in semi-automatic map drawing of complex reliefs. In section 4 the paper is concluded.

\section{THE PROPOSED SYSTEM}

The general pipeline of the algorithm is shown in Figure 1. The proposed line drawing algorithm uses two main parts: A Photometric Stereo (PS) component and a Fringe Projection (FP) scanner. As mentioned before, PS method is used to compute the surface normals for each points (Figure 1b). In this method the variation of image intensity proportional to change of light source position is used to compute surface normals (Woodham 1980). The relation between any point and its irradiance is described as (Nayar, Ikeuchi et al. 1991):

$$
\begin{aligned}
& I_{s}=K_{D} \cos \left(\varphi_{i}\right)=K_{D}\left(n \cdot L_{s}\right), \\
& K_{D}=\text { albedo } \\
& \mathrm{n}=\text { surface normal vector } \\
& L=\text { incident light direction } \\
& s=\text { index of light sources } \\
& \varphi_{i}=\text { incident angle }
\end{aligned}
$$$$
\text { where } K_{D}=\text { albedo }
$$

According to the equation (1) the normal vector for each pixel can be obtained if the light source vector (Ls) is known.

Also the FP scanner is used to obtain the object geometry (Figure 1a). The FP scanner consists of a camera and a projector with reasonable base as triangulation system. A phase-shifting fringe projection method is utilized Eq. (1) and Eq. (2) to measure the phase of each camera pixel. The absolute phase of

\footnotetext{
* Corresponding author
} 
each pixel is calculated by multiple-wavelength phase unwrapping method (Zhang 2009) and is used to establish the correspondence between camera pixels and projector pixels for stereo triangulation.

$$
\begin{aligned}
& I_{i}=A_{(x, y)}+B_{(x, y)} \cos \left(\varphi_{(x, y)}+\frac{2 \pi i}{N}\right), \quad i=1, \ldots, N \\
& \varphi_{(x, y)}=\arctan \left[\sum_{i=1}^{N} I_{i} \sin \left(\frac{2 \pi i}{N}\right) / \sum_{i=1}^{N} I_{i} \cos \left(\frac{2 \pi i}{N}\right)\right]
\end{aligned}
$$

where $\quad I_{i}=$ image intensities

$A=$ background intensity

$B=$ intensity modulation

$\varphi=$ phase value

$\delta i=$ phase shifts

The normals are needed to extract the object lines. However, as the PS images contain lens distortions and perspective effects, they cannot be used for drawings. In this paper, this is achieved through a so-called orthonormal image which is defined in the object space. On this image, instead of R,G,B, the pixels are assigned with the normal vector elements computed by the PS technique. Therefore, in addition to being geometrically correct, the measurements would not be subject to shadow, texture and other optical effects that usually come with RGB-based line extraction techniques.

As reliefs are usually complex or larger than the camera field of view, the system is placed in several stations and the image acquisition is repeated until all parts of the object are covered.
The resulting data, thus need to go through a registration process. In this paper, the common parts of the individual point clouds are used to solve for the registration parameters. Once the point clouds are registered, the ortho-normal plane is defined parallel to the relief's overall background. In addition, the registration parameters are applied to the normal data to bring them into the same coordinate system as that of the point cloud (Figure 1c).

Once the orthonormal image is formed. It can be used for extraction of object lines which is done automatically. As mentioned, to avoid usual RGB-related problems we use the normals. As, the curvature computation is performed by a differential operator (Figure 1d) high frequency noise usually present in the data is amplified. In addition, parts of ancient reliefs are usually eroded. To compensate for such problems which affects the quality of the lines extracted, the curvatures are enhanced in the end (Figure 1e). The result, are the curvilinear structures (edges), which are used as the input to the semi-automatic line drawing process.

In next stage, the edge points are extracted and linked together to from lines (Figure 1f). In the damaged areas and/or junctions, it is possible that points are linked together wrongly. Therefore, a mechanism is used to prevent the algorithm from flowing wrong lines. To do this, the radius of search area along with the previous edge direction is increased to the next starting edge point. However, they still may be small portion of the lines which may be incomplete or wrongly identified. These are edited by the user who also layers the data for further Computed Aided Design (CAD) applications (Figure 1g).

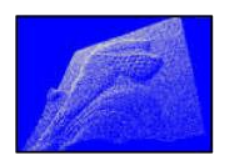

a. Scanning Object Surface by Fringe Projection Scanner
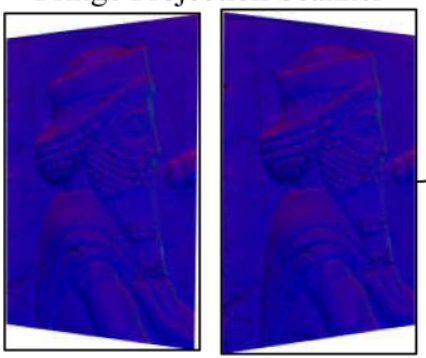

b. Computing Surface Normal by Photometric Stero

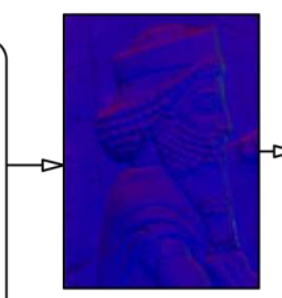

c. Geometric Correction of Normals

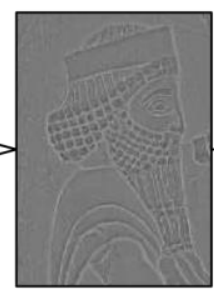

d. Computing Curvatures

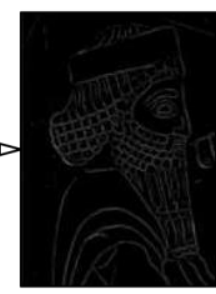

e. Enhancing Curvatures

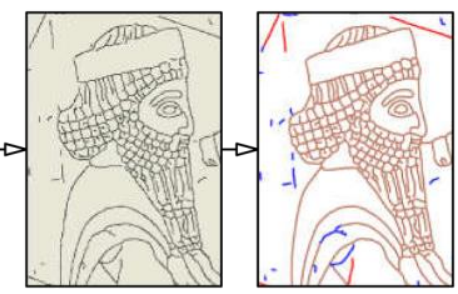

f. Tracing Curves g. Transferring to CAD

Figure 1. Pipeline of the proposed method

\section{SYSTEM EVALUATION}

In order to evaluate the proposed method, the system was implemented and two ancient reliefs were measured. The first one was from the Moghadam museum in Tehran-Iran and also the second one was in Persepolis palace in Shiraz (Figure 3). Figure 2 shows a snapshot of the proposed system. The scanner consists of a digital camera (Canon SX110) and a DLP projector (Infocus IN114), all of which are fixed on a metal base (Figure 2). The PS component is equipped with 12 LED light sources placed around the camera. The resolution of camera image is $3456 * 2592$ that it covers an area about $50 * 38 \mathrm{~cm}$. Also the pixel size on the object surface is about
$0.1 \mathrm{~mm}$. But the final produced orthonormal image has $0.2 \mathrm{~mm}$ pixel size on the object surface. As the curvature extraction is done in the $2 \mathrm{D}$ image, the overall background surface of the relief must be planar. Therefore, the proposed system is not able to measure curved reliefs. 


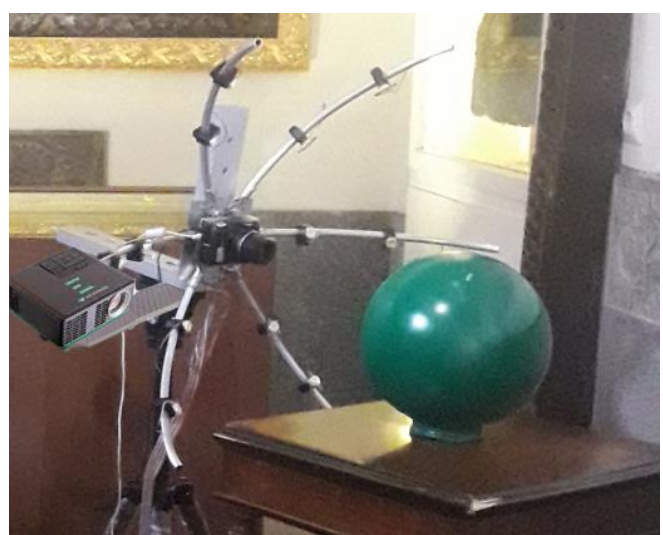

Figure 2. A view of the proposed system.

examined. Figure 3 shows the results. As can be seen, the proposed system has been drawn almost all of the relief's edges. In the following, these results are examined for visual quality and completeness. For this, an overall Orthophoto was created and the drawings were draped on it for visual inspection. Figure $3 \mathrm{c}$ shows the results. As can be seen, the drawings fit the underlying object edges very well and there is very little noise present.

To examine the completeness, the drawings of both reliefs were transferred to CAD and inspected by an operator. In this regard, the operator has drawn the incomplete drawings and finally the total length of manually drawn lines is calculated. Therefore, the overall inspection concluded that 99.1 percent of all edges for the relief of Moghadam museum and also 99.41 percent of all edges for the Bar Aam are drawn.

In the following, at first, semi-automatic drawing of the relief is done using the proposed system. Then the visual quality is

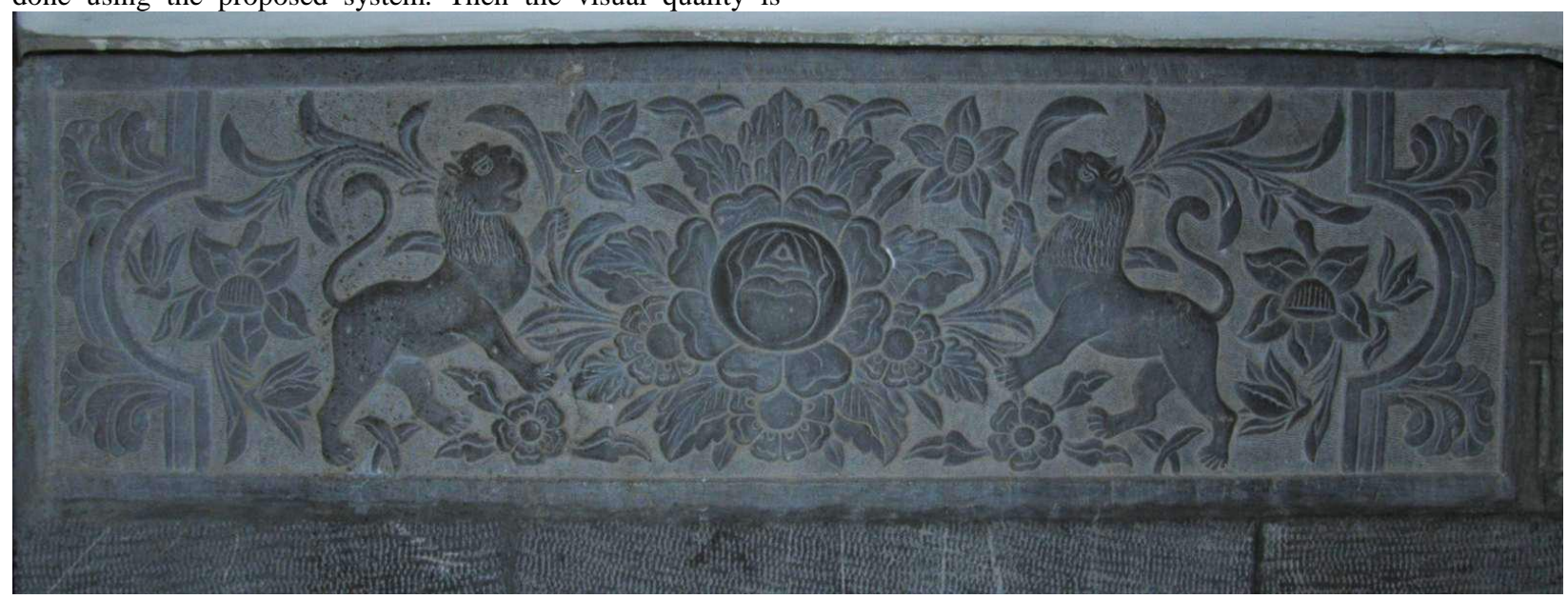

(a) 
The International Archives of the Photogrammetry, Remote Sensing and Spatial Information Sciences, Volume XLII-4/W18, 2019 GeoSpatial Conference 2019 - Joint Conferences of SMPR and GI Research, 12-14 October 2019, Karaj, Iran

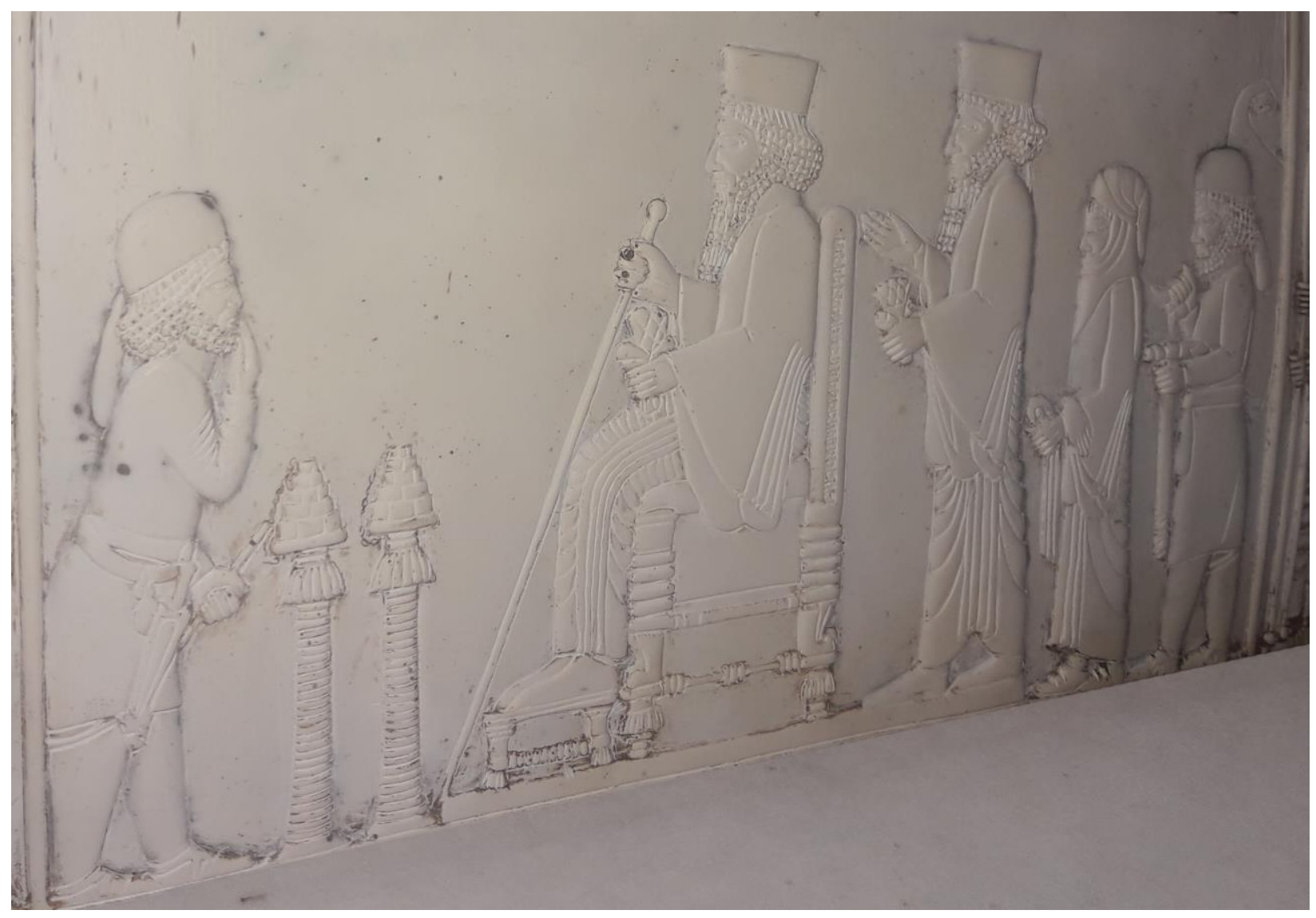

(b)

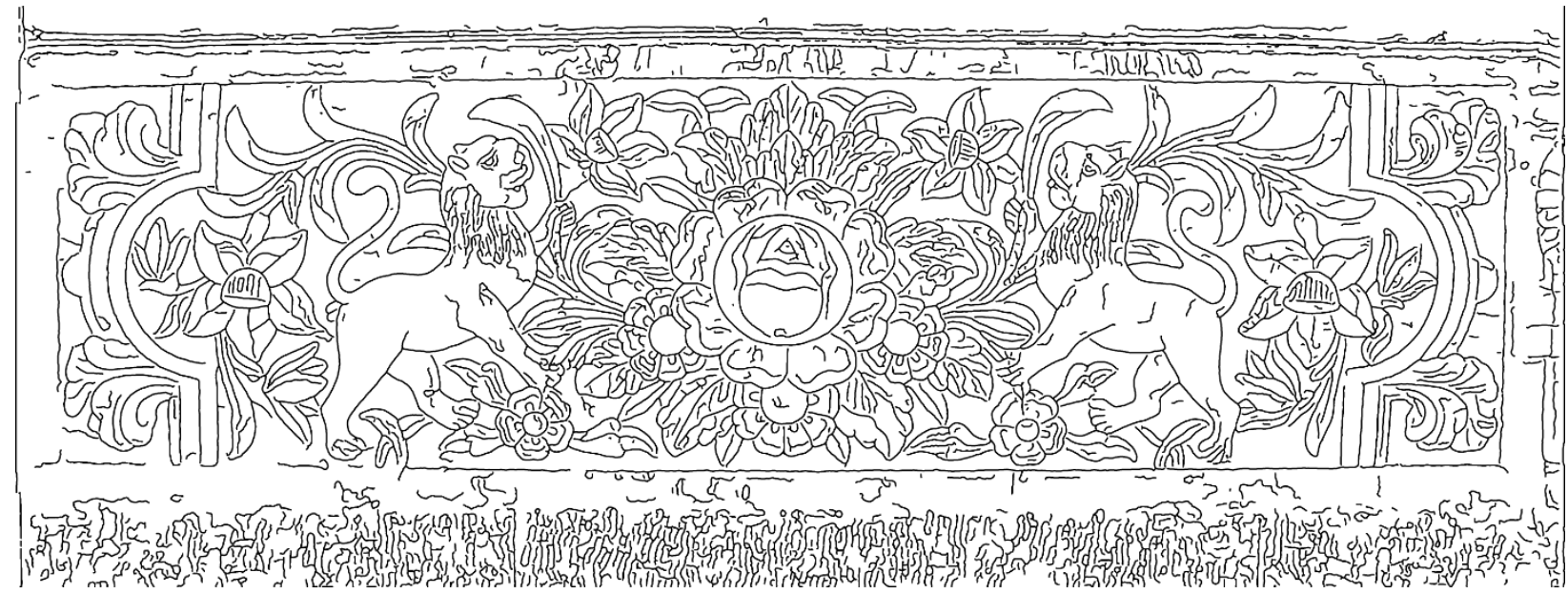

(c) 
The International Archives of the Photogrammetry, Remote Sensing and Spatial Information Sciences, Volume XLII-4/W18, 2019 GeoSpatial Conference 2019 - Joint Conferences of SMPR and GI Research, 12-14 October 2019, Karaj, Iran

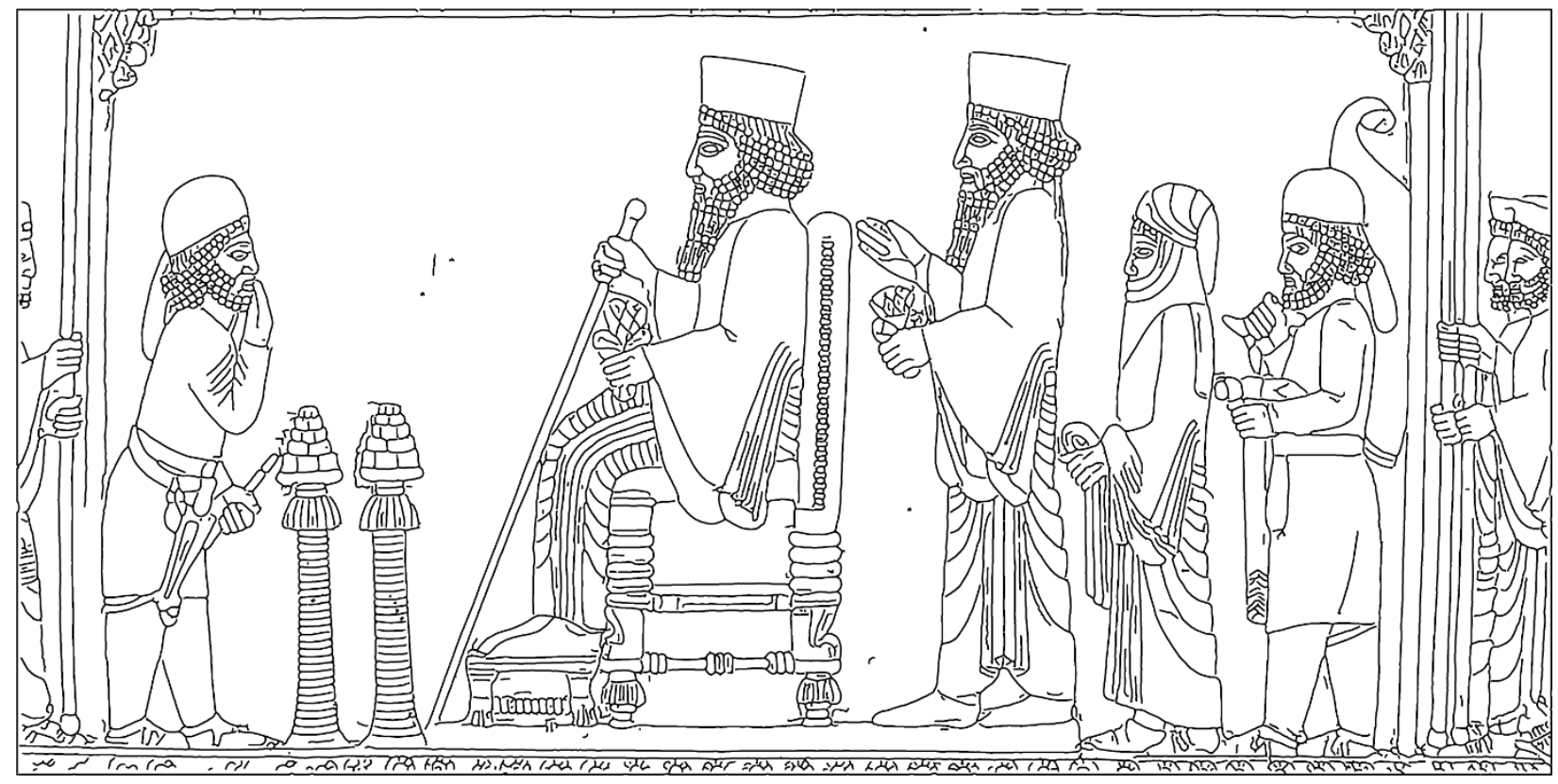

(d)

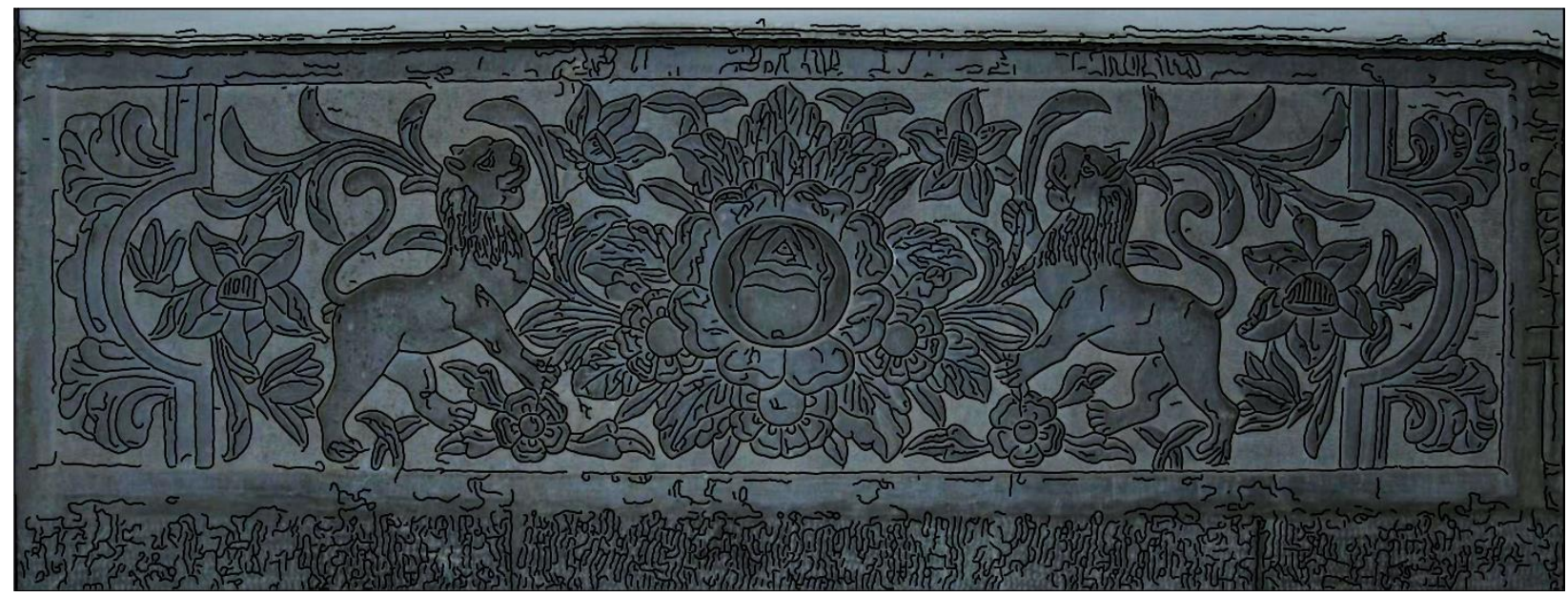

(e)

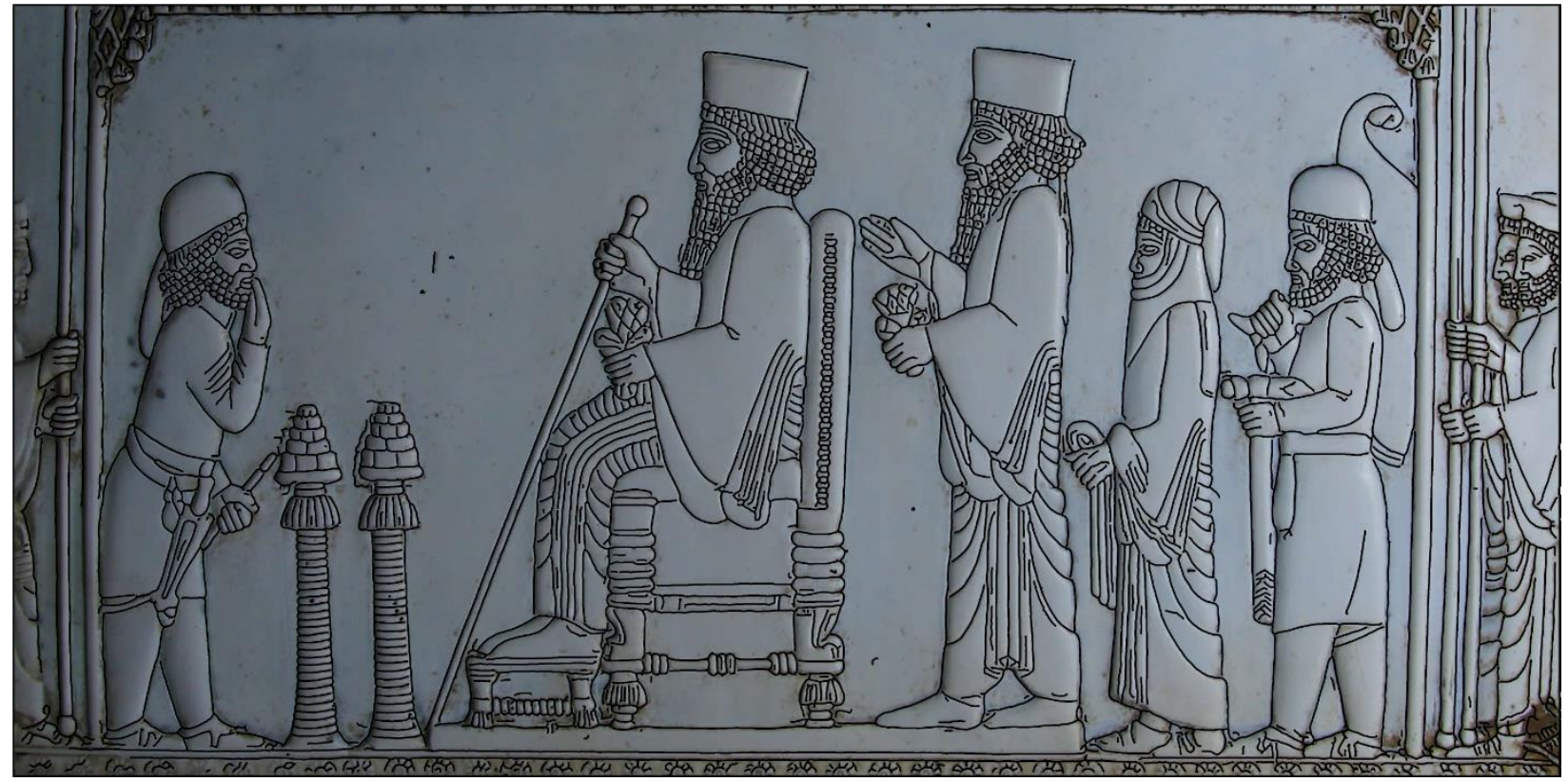


Figure 3. Evaluation of the proposed system. (a, b) Image of the anceinet relief of Moghadam museum and Bare Aam in Persepolis palace. (c, d) Semi-automatic drawing of the reliefs in $(a, b)$ by the proposed system. (e, f) Overlaying of the drawings on related orthoimages of the reliefs.

\section{CONCLUSIONS}

Line drawing is one of the main parts of a documentation report in cultural heritage. Currently most of drawings needs human interaction. In this paper we proposed a hybrid scanner to produce $2 \mathrm{D}$ maps of ancient reliefs semi-automatically. It uses photometric stereo method to provide thematic information guiding the 2D line drawing process and also uses 3D scanner to capture the geometry. The evaluation result shows that the proposed semi-automatic line drawing system can draw more than $99 \%$ of total lines in an ancient relief.

One of the limitation of the proposed system is the object dimension. If the object dimension is larger than the scan area, measurements must be made at multiple stations and then the scan data from stations are merged together. Merging point clouds and also the normal images must be challenging. Also if the overall surface of the relief has curvature, for example cylindrical stamps, this can be cause a kind of problems during curvature extraction. These are still an open issue and could be further investigated.

\section{REFERENCES}

Goesele, M., et al. (2003). Accuracy of 3D range scanners by measurement of the slanted edge modulation transfer function. Fourth International Conference on 3-D Digital Imaging and Modeling, 2003. 3DIM 2003. Proceedings., IEEE.

Karkınlı, A. E., et al. (2016). "Graphical documentation of antic relief surfaces." Journal of Cultural Heritage 21: 894-898.

Lu, Z., et al. (2013). "A 3D imaging framework based on highresolution photometric-stereo and low-resolution depth." International journal of computer vision 102(1-3): 18-32.

Morita, J., et al. (2003). "Efficient noise reduction of laser scanning data for archaeological survey." international archives of photogrammetry remote sensing and spatial information sciences 34(5/w12): 241-244.

Nayar, S. K., et al. (1991). "Shape from interreflections." International journal of computer vision 6(3): 173-195.

Nehab, D., et al. (2005). Efficiently combining positions and normals for precise 3D geometry. ACM transactions on graphics (TOG), ACM.

Pintus, R., et al. (2014). Geometric Analysis in Cultural Heritage. GCH.

Woodham, R. J. (1980). "Photometric method for determining surface orientation from multiple images." Optical engineering 19(1): 191139.

Zhang, S. (2009). "Phase unwrapping error reduction framework for a multiple-wavelength phase-shifting algorithm." Optical engineering 48(10): 105601. 